\title{
PEC Reliability in 3D E-beam DOE Nanopatterning.
}

\author{
Stanislav Krátký, Michal Urbánek, Vladimír Kolařík
}

Institute of Scientific Instruments of the AS CR, v. v. i., Brno, Czech Republic.

\section{Introduction}

Diffractive optical elements (DOE) such as binary gratings, blazed gratings, and computer generated holograms are commonly used in coherent optics (beam splitters, beam shapers, diffusers) [1]. High quality preparation of these structures is necessary to obtain designed properties. There are lots of techniques that can be used for manufacturing of such elements: engraving, etching, and various kinds of lithography (photolithography, e-beam lithography, ion lithography) [2,3]. Despite of high cost and low throughput, e-beam lithography (EBL) is a method which allows creating of fine structures in high resolution [4].

Nevertheless, it is not an easy task to prepare DOE in order to meet the requirements for coherent optics, especially in the case of multilevel and blazed ones. Required parameters are range, linearity, smoothness, homogeneity, and repeatability. Due to the scattering effects in resist material, it is hard to predict the precise shape of exposed structures after resist development. These scattering effects (forward scattering, backward scattering) are described by proximity effect causing the adjacent regions to the exposed one will receive non-zero dose [4].

Proximity effect can be expressed by the point spread function (PSF) which represents the absorbed energy distribution in radial distance from the point of incidence after point exposure [5]. The PSF are usually obtained by Monte Carlo simulation using various algorithms containing physical model of electron interactions in defined materials (mostly two layer sandwich - substrate and resist) [6]. Mathematically it can be described by double Gaussian approximation [7]:

$$
f(r)=\frac{1}{\pi(1+\eta)}\left[\frac{1}{\alpha^{2}} \exp \left(-\frac{r^{2}}{\alpha^{2}}\right)+\frac{\eta}{\beta^{2}} \exp \left(-\frac{r^{2}}{\beta^{2}}\right)\right]
$$

where $\alpha$ is the coefficient of forward scattered electrons, $\beta$ is coefficient of backscattered electrons, and $\eta$ is the ratio of the backscattered energy to the forward scattered energy. These coefficients can be also obtained by experimental methods [8]. If the PSF is known (or rather the spread coefficients), proximity effect correction (PEC) can be calculated which means that doses at each point of the exposed area are determined with respect to the range of proximity exposures. Model for PEC calculating used in this work is expressed as [9]:

$$
E_{x}=D_{x} \otimes \frac{1+\eta g_{\beta}}{1+\eta}
$$

using $E_{x}$ for absorbed energy, $D_{x}$ for exposure dose, and $g_{\beta}$ for backscatter Gaussian function.

This contribution deals with various PEC approaches on multilevel structures using BEAMER software by GenISys. We are using two different expressions of PSF - the numerical model, and the corresponding Gaussian approximation $(\alpha, \beta, \eta)-$ to calculate exposure doses. Afterwards, the simulation of real exposure distribution over the exposed area is carried out. Simulation results are 
used for reverse determination of expected depth of designed structures. Difference between designed and simulated depth helps to evaluate the reliability of various approaches for PEC.

\section{Experimental procedures}

Two inputs are essential when applying the 3D PEC in BEAMER software: the point spread function (PSF) and the contrast curve. As the PSF one can use either a numerical model or its Gaussian approximation (Figure 1). Numerical model is computed in Monte Carlo simulation software TRACER by GenISys, and it corresponds to only one layer/depth of the resist. This approach is not very precise because the PSF varies for various depths (especially for thick layer of resist which is the most often case of DOE). It would be better if BEAMER could work with 2D PSF representation (Figure 2). In the case of Gaussian approximation, parameters $\alpha, \beta, \quad \eta$ are calculated from approximation of numerical model PSF, and afterwards the mentioned parameters are used for PEC. Contrast curve (Figure 3) is entered as a table of doses and remaining resist thickness. For computing, this table is logarithmically approximated [10]. The results of PEC are relative exposure doses. Thereafter these results can be used for real exposure doses simulation of the energy absorbed in resist. There is only one input in simulation module - PSF.

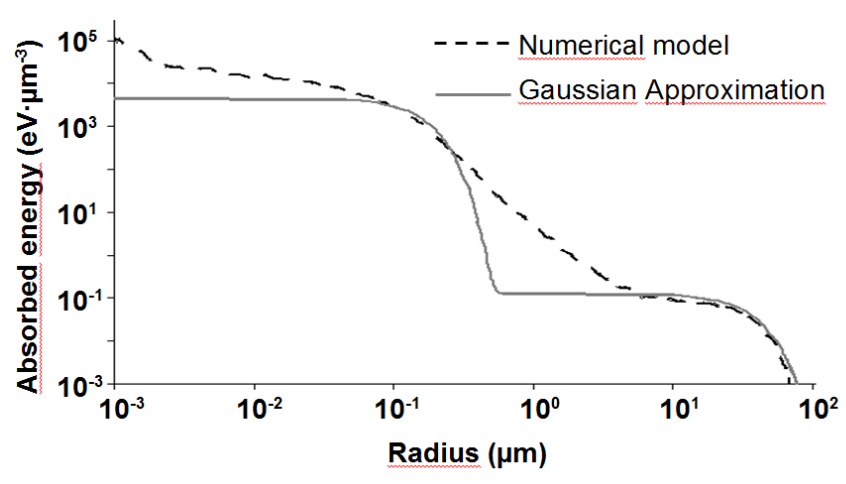

Figure 1. Numerical model of PSF, and its Gaussian approximation.

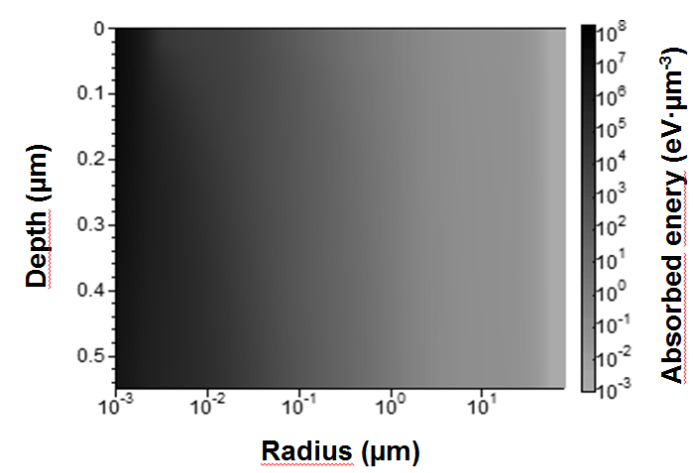

Figure 2. 2D model of absorbed energy in resist.

All simulations in this contribution were carried out for $2000 \mathrm{~nm}$ thick resist PMMA. We choose 8 level gratings with periods of 2,5 , and $10 \mu \mathrm{m}$, designed depth was always $1050 \mathrm{~nm}$ (150 nm per level). One layout was with unexposed first level and the other one was imbedded $200 \mathrm{~nm}$ under the surface of resist. Contrast curve (Figure 3) used for PEC calculations was measured on testing pattern exposed by $100 \mathrm{kV}$ e-beam system Vistec EBPG5000+ ES. The thickness of remaining resist was measured by stylus profile meter AlphaStep D120.

Contrast curve was applied in the form of polynomial (third order) approximation of measured values. The numerical model of PSF was computed in TRACER for 10 million electrons with energy of $100 \mathrm{keV}$. More precisely, we used the PSF corresponded almost to the maximum depth of resist layer ( $25 \mathrm{~nm}$ above the substrate surface). PEC was also calculated with Gaussian approximation of chosen PSF. The same PSF model (the both numerical one and its approximation) was used for subsequent simulations of absorbed energy. 


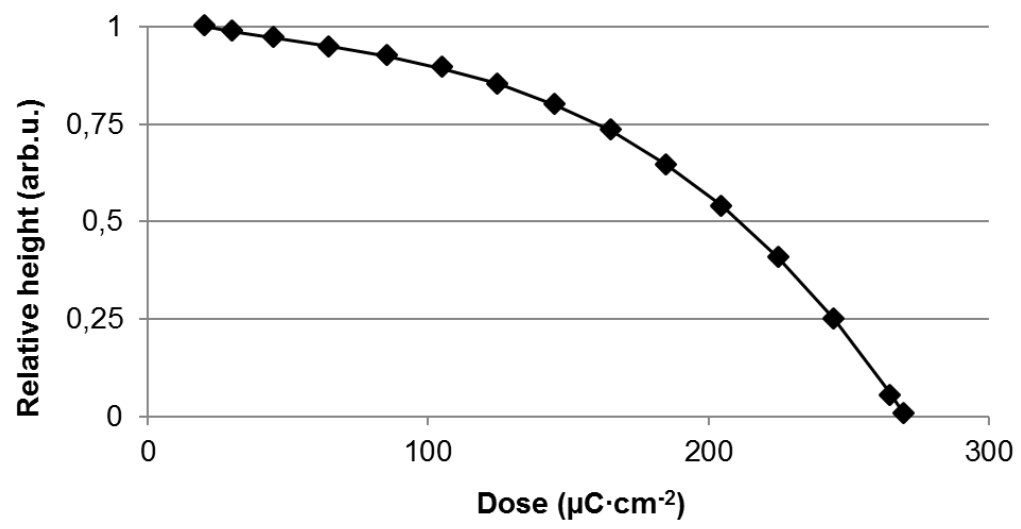

Figure 3. Contrast curve of PMMA $2000 \mathrm{~nm}$, non-alcoholic developer.

\section{Results}

All results will be demonstrated on grating with period of $10 \mu \mathrm{m}$ (the gratings with other periods had very similar results). The remaining resist thickness for each level of grating was calculated from simulated absorbed energies according to expression:

$$
h_{\text {rem }}=1.32 \cdot 10^{-4} D_{z}^{3}+1.88 \cdot 10^{-2} D_{z}^{2}-3.12 D_{z}+2.09
$$

using $h_{\text {rem }}$ for the height of remaining resist, and $D_{s}$ for absorbed energy (real dose over the area).

Firstly, we focus on comparison between layout with $1^{\text {st }}$ level unexposed, and the layout which was imbedded into the resist layer. The comparison of these two layouts for the PSF represented by Gaussian approximation, and their designs are shown in Figure 4 and Figure 5.

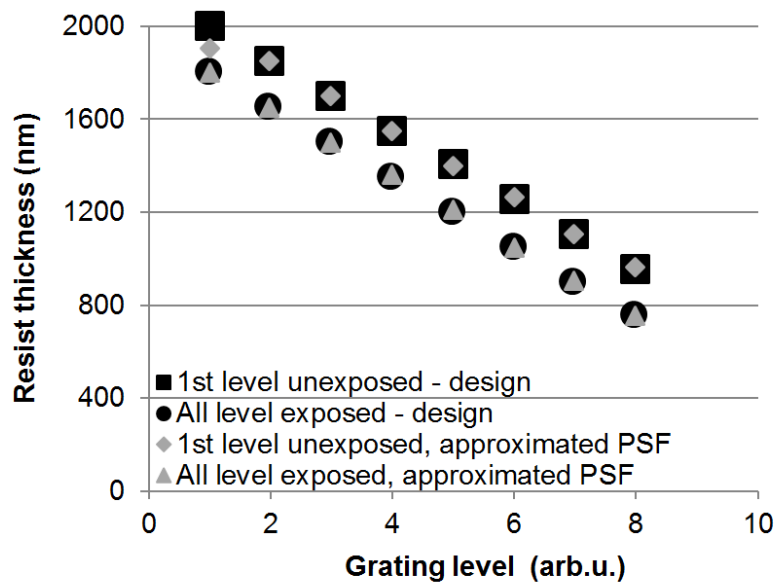

Figure 4. Comparison between the imbedded layout and the layout with $1^{\text {st }}$ level unexposed, and their designs - Gaussian approximation PSF resist thickness.

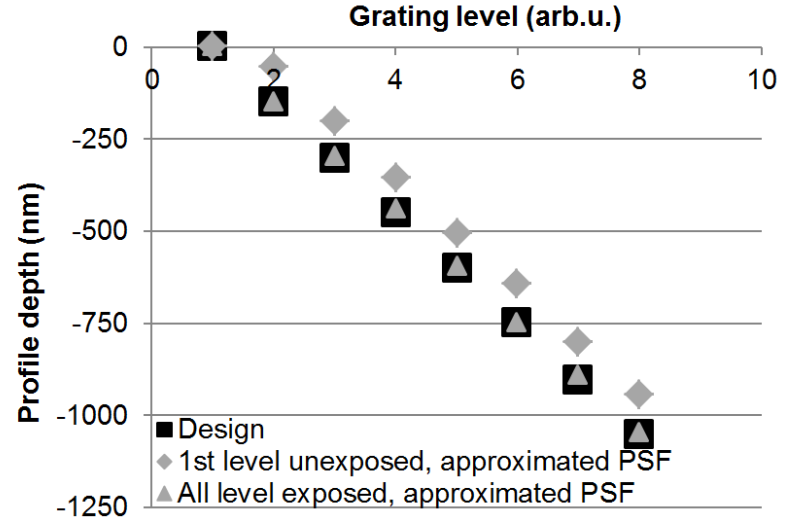

Figure 5. Comparison between the imbedded layout and the layout with $1^{\text {st }}$ level unexposed, and their designs - Gaussian approximation PSF - profile depth.

When we relate the designed depth of each level to the resist surface it corresponds very well except for the $1^{\text {st }}$ level which meant to be unexposed in one of the layouts (Figure 4). It simulates correctly that 
even the unexposed area (if it is in the range of forward scattered and backscattered electrons) received non-zero dose. Consequently, this area is also developed. However, if we evaluate the depth of grating profile (Figure 5), the result of layout with $1^{\text {st }}$ level unexposed is incorrect. More precisely each level is offset by the first level depth (unexposed one). That is of course critical flaw with respect to design of DOE.

If we compare the same layouts, but the ones where PEC was computed with numerical model of PSF, the results are slightly different (Figure 6 and Figure 7).

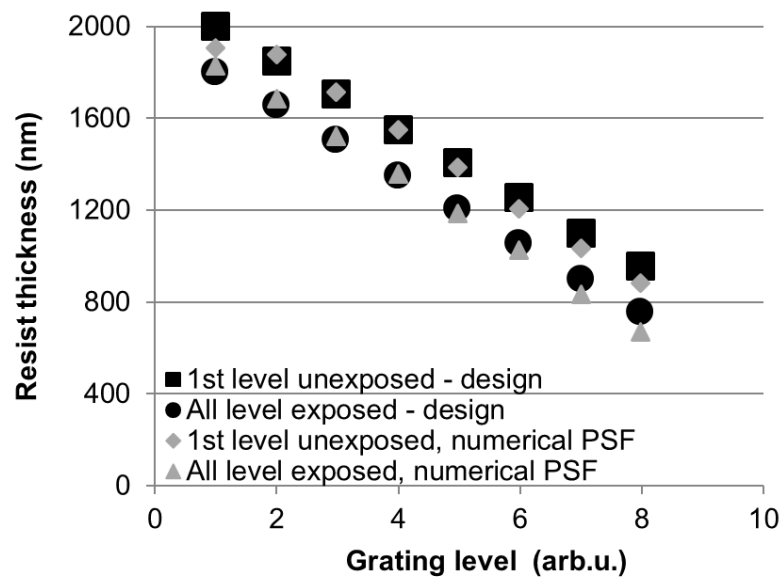

Figure 6. Comparison between the imbedded layout and the layout with $1^{\text {st }}$ level unexposed, and their designs - numerical model PSF - resist thickness.

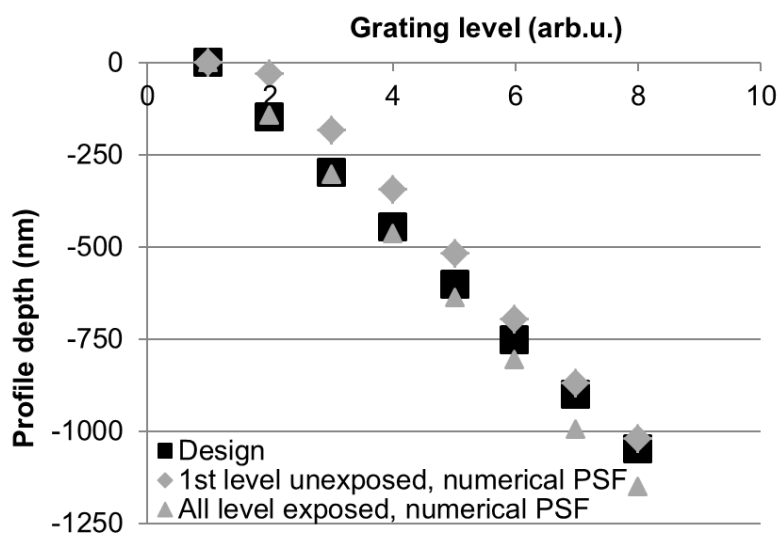

Figure 7. Comparison between the imbedded layout and the layout with $1^{\text {st }}$ level unexposed, and their designs - numerical model PSF - profile depth.

In the case of designed depth of each level in relation with resist surface, we can see deeper levels not completely matching the design for both of the layouts (Figure 6). The method using Gaussian approximation is more accurate than the one with numerical model of PSF from this point of view. When we relate the depth of each level to its designed depth (Figure 7) the results are in favor to method using numerical model of PSF (in the case of layout with the $1^{\text {st }}$ level unexposed). Upper levels are also incorrect but lower ones seem to be calculated correctly. On the contrary, upper levels are computed correctly but lower ones are incorrect for the layout with all levels exposed.

Although the method with numerical model of PSF seems to be suited for layouts with intentionally unexposed areas, this claim falls when we use different PSF - different layer of resist. As it shown in Figure 8 and Figure 9, the results are incorrect neither in one case.

We would recommend using 3D PEC correction in BEAMER software only for completely exposed patterns. It is more suitable to use Gaussian approximation model of PSF than the corresponding numerical model, especially for thicker resist layers. It is also clear there will be always small area (the range of scattering effects - tens of micrometers) around the pattern which will be exposed/developed incorrectly. It is the consequence of wrong PEC calculations over the areas that are not completely exposed. 


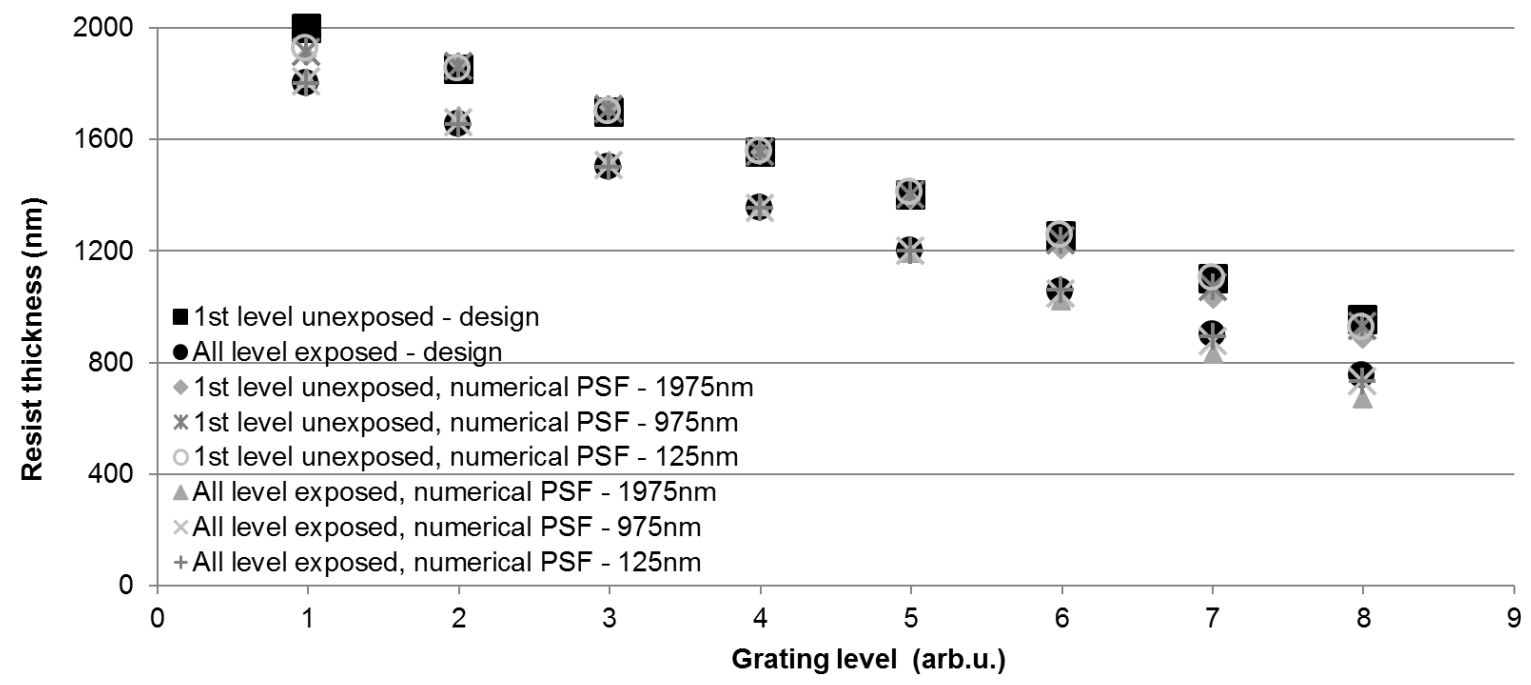

Figure 8. Comparison between the imbedded layout and the layout with $1^{\text {st }}$ level unexposed, and their designs - various layer of numerical model of PSF - resist thickness.

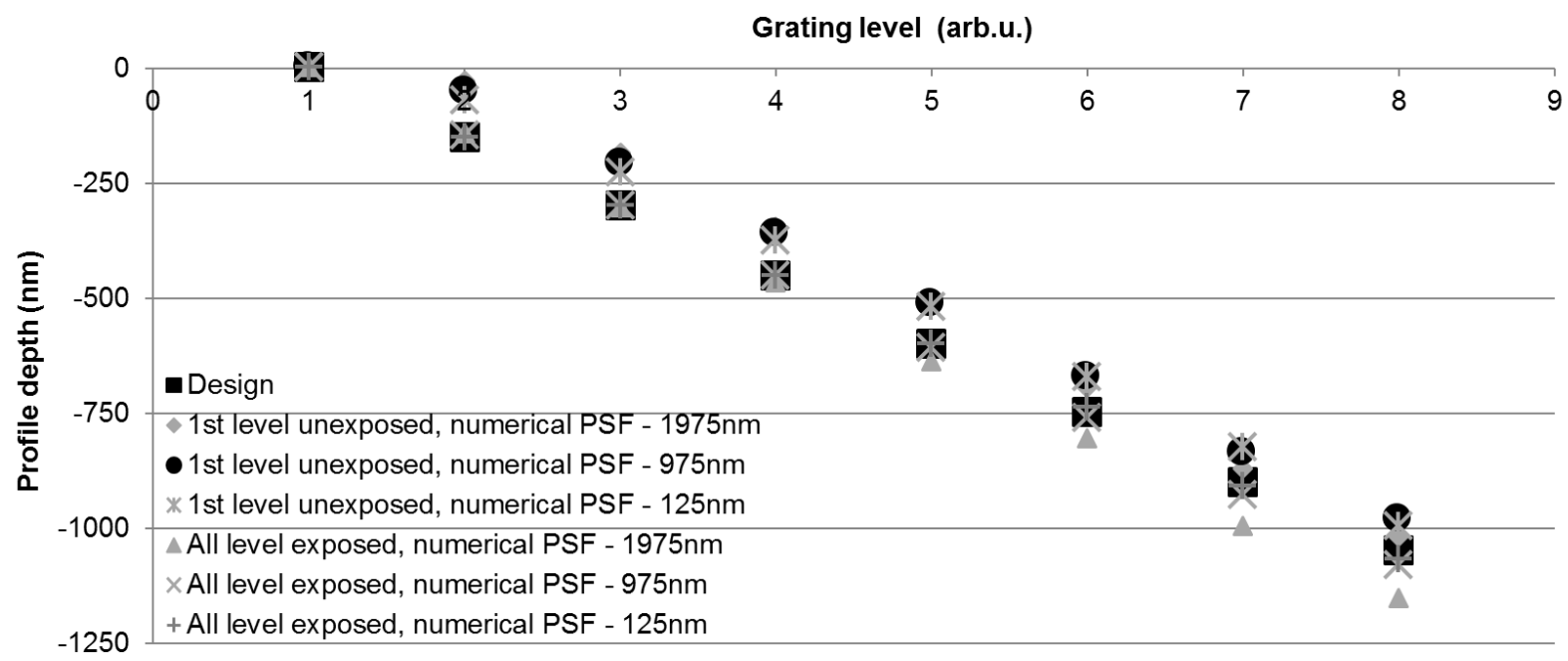

Figure 9. Comparison between the imbedded layout and the layout with $1^{\text {st }}$ level unexposed, and their designs - various layer of numerical model of PSF - profile depth.

\section{Conclusions}

We presented the reliability of PEC calculation for multilevel gratings in software BEAMER by GenISys. To obtain better results, it is recommended to use Gaussian approximation of PSF than its numerical model (at least for a thicker resist). It is also clear that it is necessary to prepare design with the whole area exposed. If there is a part of area intended not to be exposed, PEC is computed incorrectly because the exposure doses (or designed levels depths) are related to the top of resist layer. Of course, ideal simulations are usually different from real exposures. Especially when it is essential to maintain the same conditions as they were during the preparation of contrast curve (resist layer preparation, exposure, and development process). As a next step, we would like to carry out the exposure where we could verify the results from described simulations [10]. 
References:

[1] DC O'Shea et al in “Diffractive Optics: Design, Fabrication, and Test”, (SPIE, Washington) p. 1.

[2] Z Cui in "Nanofabrication Principles, Capabilities and Limits", (Springer, New York) p. 1.

[3] EG Loewen et al in "Diffraction Gratings and Applications", (Marcel Dekker, New York) p. 495.

[4] MA McCord and MJ Rooks in "Handbook of Microlithography, Micromachinning, and

Microfabrication, Vol. 1: Microlithography”, ed. P Rai-Choudhury, (SPIE, Washington) p. 139.

[5] M Hauptmann et al, Microelectron. Eng. 86 (2009), p. 539.

[6] S Babin et al, Microelectron Eng. 57-58 (2001), p. 343.

[7] L Harafuji et al, J. Vac. Sci.Technol. B 10 (1992), p. 133.

[8] E Seo et al, Microelectron. Eng. 50 (2000), p. 305.

[9] N Unal et al, Microelectron Eng. 87 (2010), p. 940.

[10] The authors acknowledge funding from MEYS CR (project No. LO1212), the TACR project No. TE 01020233 and by the institutional support RVO: 68081731. 